\title{
Screening of Cotton Genotypes for Protein Content, Oil and Fatty Acid Composition
}

\author{
Yuksel Bolek ${ }^{1}$, Halil Tekerek ${ }^{1}$, Khezir Hayat ${ }^{2} \&$ Adem Bardak $^{1}$ \\ ${ }^{1}$ Department of Agricultural Biotechnology, Faculty of Agriculture, Kahramanmaras Sutcu Imam University, \\ Kahramanmaras, Turkey \\ ${ }^{2}$ Department of Bioengineering \& Sciences, Faculty of Agriculture, Kahramanmaras Sutcu Imam University, \\ Kahramanmaras, Turkey \\ Correspondence: Yuksel Bolek, Department of Agricultural Biotechnology, Faculty of Agriculture, \\ Kahramanmaras Sutcu Imam University, Kahramanmaras 46100, Turkey. Tel: 90-(344)-280-2133. E-mail: \\ yuksel@ksu.edu.tr
}

Received: February 16, 2016 Accepted: March 19, 2016 Online Published: April 15, 2016

doi:10.5539/jas.v8n5p107 URL: http://dx.doi.org/10.5539/jas.v8n5p107

\begin{abstract}
The increase in the population at the global level necessitates to explore promising approaches to increase food supply, including protein and oil, to meet the needs of the people. Cotton is one of the most important oil producing crops and cottonseed meal provides important protein nutrients as animal feed. However, information on the genetic basis of cottonseed oil and protein contents is lacking. In this study; protein contents, oil and fatty acid composition of 124 cotton genotypes were observed for developing new cultivars. Accelerated Solvent Extraction method used for determining fat ratio; Gas Chromatography employed for fatty acid analysis while protein contents were analyzed by Kjeldahl method. Average crude oil 31.0\%, total fat contents varied from 23.11 to $37.70 \%$ while mean protein content $38.0 \%$ were observed among genotypes. The dominating fatty acids included linoleic acid, palmitic acid and oleic acid (46.91, 25.73 and 20.21\%) respectively, while linolenic acid $(0.13 \%), \gamma$-linolenic $(0.33 \%)$, palmitoleic acid $(0.64 \%)$, myristic acid $(0.88 \%)$, nervonic acid $(1 \%)$ and stearic acid $(2.38 \%)$ had variations in fatty acid contents. Frequency distribution of the parameters showed a normal distribution and differences among genotypes for the traits studied were statistically highly significant. Prinicipal component analysis showed a complex opposite relationship with a total protein and oil contents. Genotypes; Fantom for protein, Cirpan 60 for total crude oil, Stoneville 468 and YB195 for higher amount of fatty acids especially oleic acid; can be used for improvement of cottonseed quality in breeding programs.
\end{abstract}

Keywords: genotype, cottonseed, nutritional value, oil, protein, fatty acids

\section{Introduction}

Cotton belongs to genus Gossypium and Malvaceae family that grows naturally as a perennial, but for commercial purposes is grown as an annual crop (Wakelyn \& Wan, 2003). Cotton is a major crop in the world (Yu et al., 2012). The five largest cottonseed producing countries (China 26.4\%, India 25.8\%, USA 10.7\%, Pakistan $7.9 \%$, Brazil $6.5 \%$ ) currently account for $77.3 \%$ of global output (ICAC, 2015). Cotton fiber is a source of natural textile, and cottonseed is a source of oil for human consumption, cotton meal and minerals for livestock feed (Yu et al., 2012; He et al., 2013). Therefore, maintaining high quality fiber and cottonseed nutritional value is critical. Cottonseed is the second major product from the cotton plant (after fiber) which serves as raw material for oil extraction or animal feed production (Hamilton et al., 2004; Ashokkumar \& Ravikesavan, 2008).

Cottonseed oil is among the most unsaturated oils, others being safflower, corn, soybean, rapeseed and sunflower seed oils. Oil cakes/oil meals are by-products obtained after oil extraction from the seeds. Oil cakes are of two types, edible and non-edible. Edible oil cakes have a high nutritional value; especially have protein contents ranging from $15 \%$ to $50 \%$ (Ramachandran et al., 2007). Cottonseed oil has a ratio of $2: 1$ of polyunsaturated to saturated fatty acids and generally consists of $65-70 \%$ unsaturated fatty acids including $18-24 \%$ monounsaturated (oleic) and $42-52 \%$ polyunsaturated (linoleic) and $26-35 \%$ saturated (palmitic and stearic) (Agarwal et al., 2003). Cottonseed oil performs better than other oil as it lasts a long time and stores well by withstanding higher temperature for food items due to its high antioxidant content (Sekhar \& Rao, 2011). 
Recently, cotton has attracted much attention as a biofuel crop (Chesterfield et al., 2013), however, information on the genetic basis of cottonseed oil and protein contents is lacking (Jiwen et al., 2012). For improving the quantity and quality of cottonseed oil content, various breeding procedures have been employed with different levels of success (Cherry et al., 1981; Dani, 1990). Many varieties of cotton have been developed with improved crop yield and productivity (Calhoun \& Bowman, 1999). Breeding cottonseed for oil content has depended mainly on phenotypic information that is used to select varieties with high seed oil content (Azhar \& Ahmad, 2000; Ash \& Dohlman, 2006; Pahlavni et al., 2008). The availability of genetic variation effects the outcome of a breeding program (Cruz \& Carneiro, 2003; Silveria, 2007). Dani (1989, 1991), Gotmare et al. (2004), Khan et al. (2009) showed significant variation of oil contents in cotton. In analytical chemistry, isolation from solid samples (such as Soxhlet extraction, flask extraction) are tedious and time-consuming, which lowers the sample throughput. Accelerated Solvent Extraction is a fully automated technique, Supercritical Fluid Extraction is less solvent and have the power to make the extraction in a faster time (Hana et al., 2001).

Cottonseed oil and the high protein content are important features, especially because of the use as animal feed and human food. While developing new cotton varieties, fibers are always considered of added value. For this purpose, the prevailing germplasm, consisting of 124 genotypes and having diverse features will be screened for protein, oil and fatty acids contents. The ultimate objective is to select the best genotypes having desired traits.

\section{Material and Methods}

\subsection{Field Trial and Collection of Samples}

The germplasm having different origins were selected from three different species Gossypium hirsutum L. (114), G. barbadense L. (9) and G. herbaceum L. (1). Genetic stock was planted in field during 2012 by keeping planting distance $(\mathrm{L} \times \mathrm{W})$ by $20 \times 70 \mathrm{~cm}$. The experiment conducted as a randomized complete block. Normal agronomic practices were applied and plant protection measures carried out and 50-boll sample harvested from each row and ginned to recover fiber and fuzzy seed. Fifteen grams of fuzzy seed was then subsampled for determination of fatty acid composition. All the genotypes screened for protein, oil content and fatty acid contents.

\subsection{Sample Preparation}

The cottonseed of genotypes delinted by hand and 100 seed weight measured on a precision scale. The seed coat and embryo separated from each other. Cottonseed cores were transfered into $15 \mathrm{ml}$ falcon tube and were stored at $4{ }^{\circ} \mathrm{C}$. The rudimentary seeds were put in oven at $103{ }^{\circ} \mathrm{C}$ and dried for $2 \mathrm{hr}$. Seeds were put in dessicator for 30 min, cooled and dehumidified; the grinded seeds were again put in the dessicator to avoid moisture loss. Silica jel was kept in dessicator to keep samples wet.

\subsection{Total Fat Analysis}

About $1 \mathrm{~g}$ of the sample in powder form was weighed for ASE (Accelerated Solvent Extraction) (Abdelmoez et al., 2011). ASE device works good with high purity nitrogen. The solvent used as $n$-hexane. The optimum operating temperature for $\mathrm{n}$-hexane is $80^{\circ} \mathrm{C}$ because device is heated to furnace temperature and $\mathrm{n}$-hexane flows in the samples through vials. After passing the samples for $30 \mathrm{~min}$ the oil is provided by the extract. Extraction from the sample is finished through liquid n-hexane. The oil is obtained at the bottom of the device in a collecting pot. The resulting extracted sample flasks transfered to evaporator for evaporation. For this flask should be fixed and weighing must be done. Flask are kept for $1 \mathrm{~h}$ then heated at $103{ }^{\circ} \mathrm{C}$ for half an hour in a desiccator and allowed to cool. This procedure was repeated 3 times.

\subsection{Fatty Acid Extraction}

Extracted samples were measured as tear weight before being placed in empty flasks. Samples obtained by extraction to constant weight was transfered from the flask. In evaporator at $80{ }^{\circ} \mathrm{C} n$-hexane had been evaporated from the flask; it takes about 15-minutes. This process is completed in flask, water remaining in the flask may be volatile for evaporation; flasks were again put in the oven at $103{ }^{\circ} \mathrm{C}$ for $1 \mathrm{hr}$, and then placed in the dessicator for $30 \mathrm{~min}$ to remove moisture and then allowed to cool down. After completion of this process weighing carried out, total percentage of fat obtained from $1 \mathrm{~g}$ is calculated. Oil contents were measured by following the fomula (Karahan, 2007).

$$
\text { Oil Contents }(Y M)=a / M(g) \times 100
$$

Where,

YM: Oil content (\%); a: Fat amount accumulated in flask (g); M: sample weight (g). 


\subsection{Fatty Acid Analysis}

$0.1 \mathrm{~g}$ (approximately 2-3 drops) of oil extracted from the cottonseed were transferred to $15 \mathrm{ml}$ glass tube. Upon addition of $10 \mathrm{ml} \mathrm{n}$-hexane the tube capped and vigorously shaked. On this, $0.5 \mathrm{ml}$ of $2 \mathrm{~N}$ methanolic $\mathrm{KOH}$ added and shaking repeated. The tube heated for 1-2 hours in a dark environment untill the clear supernatant is obtained. Supernant was accumulated in the upper part and placed in $2 \mathrm{ml}$ vials which is ready for GC analysis.

\subsection{Fatty Acids Analysis of GC}

Standards available in 37 different fatty acids in Fame 37 standard mix were used and the percentage of these contents is known. After entering the amounts of standards, loaded into the device and explained by different parameters. We injected the samples and the fatty acid \% was determined. After methyl estrification, the samples were transfered in to $2 \mathrm{ml}$ vials and placed on the device. $1 \mu \mathrm{l}$ sample was injected into the injection port at $240{ }^{\circ} \mathrm{C}$. The device sent it to column via carrier phase by dividing $1 / 100$ and $177 \mathrm{kp}$ pressure (TRCN 100 Techno Chroma). Samples at column cooled down to $70{ }^{\circ} \mathrm{C}$ then started to increase again up to $240{ }^{\circ} \mathrm{C}, 2{ }^{\circ} \mathrm{C}$ increase for every 5 minutes. With this program which has been translated in accordance to methyl-ester and proceed by separating the different fatty acids in the column on molecular size. The order to leave the coloumn is according to detector values. Detector (FID-flame ionization detector) burn again to $250{ }^{\circ} \mathrm{C}$ to decompose the ions. Solvent is leaving first in detector. According to the ion intensity signal on the chromatogram baseline peak is created for the analysis of fatty acids.

\subsection{Protein Analysis}

Protein analysis was performed according to Kjheldahl method. Cottonseed grounded to make a homogenous powder by using micro mill. $1 \mathrm{~g}$ of powdered sample was weighed and placed in the Kjeldahl combustion tube. $25 \mathrm{ml}$ of sulfuric acid (95-98\%) and 2 catalyst tablets were added. Kjeldahl tube was placed in combustion unit and burned to $420{ }^{\circ} \mathrm{C}$ for $60 \mathrm{~min}$. After burning untill the color of the solution becomes clear light greenish burning repaeated for more than 30 minutes. Once this is finished, the tube was cooled to room temperature prior to combustion. The tubes were cooled after wet digestion and placed in wet distillation unit. $25 \mathrm{ml}$ boric acid added in the flask and placed at bottom of ammonia collecting unit. The device automatically added $100 \mathrm{ml}$ of $35 \mathrm{NaOH}$ (\%) (sodium hydroxide). The sample added to Kjeldahl tube and flask is left in ammonia collection unit for 5-minutes. The reading was observed by placing the sample tube in a non distilled unit. 5-6 drops of methylene was dropped in flask and distillate collected as methylene blue-methylene red. It was treated to stock solution $0.2 \mathrm{~N} \mathrm{HCl}$ (hydrochloric acid) with the automatic burette. Dissipated $\mathrm{HCl}$ will be saved as a solution. Results are calculated by using following formula:

$$
\mathrm{N} \%=\frac{(\mathrm{V} 2-\mathrm{V} 1) \times \mathrm{N} \times 0.014 \times 100}{\mathrm{~m}}
$$

Where,

$\mathrm{V} 2$ = Initial dissipated $0.2 \mathrm{~N} \mathrm{HCl}$;

$\mathrm{V} 1=$ Unsighted solution $0.2 \mathrm{~N} \mathrm{HCl}$;

$\mathrm{N}=\mathrm{HCl}$ normality;

$\mathrm{m}=$ Sample quantity $(\mathrm{g})$;

$0.014=$ The equivalent weight of nitrogen.

The total amount of nitrogen found by this formula and the amount of protein was determined by using an other formula: Protein $(\%)=\mathrm{N}(\%) \times 6.25$ (conversion factor).

\subsection{Statistical Analysis}

All the data were subjected to analysis of variance and variability in fatty acid composition was measured by SAS (v9) and compared by the Tukey's test. Frequency distribution of genotypes done and graphical analysis of the components from subset of genotypes illustrates a number of points regarding differences in the effects of genotype observed in whole dataset (Figure 3). The width of the component bands in these plots reflects a measure of the variation due to genetics. Moreover; elite genotypes screened on the basis of protein contents and other nutritional values. From measured distributions, a number of characteristic fatty acid parameters were also calculated. The principal component analysis and correlation analysis was conducted among genotypes to identify relationships among observed traits. 


\section{Results}

Relative values for the means, standard deviations and the range of oil, protein contents and individual fatty acid compositions of the genotypes were shown in Table 1. The diverse sources of the samples contributed to the high variability in the sample population. Analysis of variance showed significant $(\mathrm{p}<0.01)$ differences among all genotypes for means of 100 seed weight (g), protein contents, oil contents, and all fatty acids icluding, palmitic acid, stearic acid, palmitoleic acid, oleic acid, nervonic acid, linoleic acid, and $\gamma$ - linolenic acid contents among genotypes while non-significant differences were observed for myristic acid and linolenic acid (Table 1). The descriptive statistics for all traits of genotypes showed that the genotypes are having differences for coeficient of variability (CV\%). Moreover; Tukey test among genotypes used for estimating the mean values of accessions observed for desirable contents in commercial point of view (Table 2). The concentration and scale of variability in oil and fatty acid contents of the samples used in this study are similar to those reported by other authors (Hamilton et al., 2004; Nyanzi et al., 2005; Dowd et al., 2010). Considering the values for all the traits, the variability ranged from 2.32 to $70.6 \%$ in the germplasm indicating obvious differences within the population. The widest ranges were observed for oleic acid, linoleic acid, palmitic acid, oil and protein contents. Variability within the population as shown by the standard deviation values ranged between 0.1 and $2.96 \%$ in the genotypes. The highest variability (CV\%) was observed for linolenic acid, palmitic acid, stearic acid, palmitoleic acid and $\gamma$-linolenic acid while linoleic acid, myristic acid, oleic acid, nervonic acid, oil and protein contents had the lowest values for variability.

The average value for 100 seed weight was $10.19 \mathrm{~g}$. The values varied from 7.91 to $13.92 \mathrm{~g}$ (Table 1). Genotypes having the highest 100 seed weight include AK-4 (13.92 g), Delcerro (13.52 g) and Acala Maxa (13.50 g) while the lowest value of seed weight were found Okra-Frego (7.91 g), Fantom (8.39 g) (Table 2). Cottonseed oil and protein contents are quantitative traits and both are usually negatively correlated with one another (Hanny et al., 1978; Wu et al., 2009). Anonymous (2006) observed the protein content $19.4 \%$ and $30.3 \%$ of shelled and un-shelled cottonseed, respectively. Mean protein content were 38\% among genotypes (Table 1). The genotypes varied between 34.17 and 46.33\%. High protein content observed in Fantom (46.33\%), YB 195 (43.08\%) and Acala Maxa (42.40\%) while Paymaster 2379 had least protein content (34.17\%) (Table 2). This may be due to variation of different genotypes having different origin. Great genetic variations among cotton species and cultivars in respect to cottonseed oil (17-27\%) and protein (12-32\%) contents also exist (Kohel, 1980; Kohel et al., 1985; Wu et al., 2009; Dowd et al., 2010). Quampah (2012) revealed the amount of oil inside the seeds ranged between 22.68 to $36.83 \%$ and the average amount was $31.42 \%$ while average oil content of genotypes in our study was $38 \%$ which is inaccordance to earlier studies. The oil contents found 23.11 to $37.70 \%$ among genotypes (Table 1). The highest amount of oil contents found in Cirpan 60 (37.70\%) and the lowest amount of oil content of the genotypes include Fantom (23.11\%) and Garant (26.29\%) (Table 2). The genotype having high protein showed low oil contents are in accordance to Alkaya (2010).

Table 1. Descriptive statistics and analysis of variance for oil, protein and fatty acid contents among genotypes

\begin{tabular}{|c|c|c|c|c|c|c|c|}
\hline \multirow{2}{*}{ Traits } & \multirow{2}{*}{ Mean } & \multirow{2}{*}{ C.V\% } & \multirow{2}{*}{ Min. } & \multirow{2}{*}{ Max. } & \multicolumn{2}{|c|}{ Mean Squares } & \multirow{2}{*}{$\mathbf{F}$} \\
\hline & & & & & Genotype $(\mathrm{DF}=123)$ & Error $(D F=124)$ & \\
\hline 100- seed wight & $10.19 \pm 1.21$ & 6.9 & 7.91 & 13.92 & 2.92 & 0.49 & $5.92 * *$ \\
\hline Protein & $38.0 \pm 2.06$ & 2.32 & 34.16 & 46.33 & 7.71 & 0.78 & $9.85 * *$ \\
\hline Oil contents & $31.0 \pm 2.47$ & 5.22 & 23.11 & 37.7 & 9.68 & 2.6 & $3.71 * *$ \\
\hline Myristic Acid & $0.88 \pm 0.13$ & 2.42 & 0.48 & 1.59 & 0.033 & 0.005 & 6.11 \\
\hline Palmitic Acid & $25.72 \pm 1.54$ & 22.6 & 20.97 & 29.37 & 4.29 & 0.45 & $9.54 * *$ \\
\hline Stearic Acid & $2.38 \pm 0.44$ & 13.71 & 0.81 & 3.97 & 0.29 & 0.1 & $2.71 * *$ \\
\hline Palmitoleic Acid & $0.64 \pm 0.11$ & 12.27 & 0.44 & 1.22 & 0.022 & 0.006 & $3.64 * *$ \\
\hline Oleic Acid & $20.21 \pm 2.68$ & 3.88 & 14.39 & 33.15 & 13.88 & 0.61 & $22.51 * *$ \\
\hline Nervonic Acid & $1 \pm 0.2$ & 8.1 & 0.71 & 2.71 & 0.076 & 0.006 & $11.54 * *$ \\
\hline Linoleic Acid & $46.81 \pm 2.96$ & 2.59 & 37.84 & 54.2 & 16.1 & 1.47 & $10.94 * *$ \\
\hline Linolenic Acid & $0.13 \pm 0.1$ & 70.6 & 0.02 & 0.71 & 0.014 & 0.008 & 1.66 \\
\hline$\gamma$-Linolenic Acid & $0.33 \pm 0.06$ & 14.93 & 0.12 & 0.69 & 0.006 & 0.002 & $2.65 * *$ \\
\hline
\end{tabular}

Note. ${ }^{*}: \mathrm{p}<0.01$. 
Table 2. Protein, oil and fatty acid contents of selected genotypes by Tukey test

\begin{tabular}{|c|c|c|c|c|c|c|c|c|c|c|c|c|}
\hline 芯 & 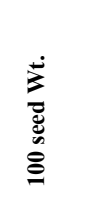 & 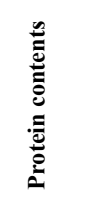 & 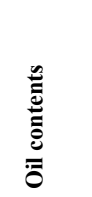 & 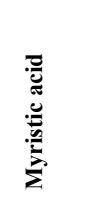 & 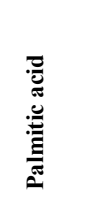 & 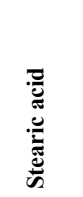 & 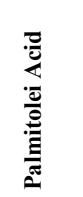 & 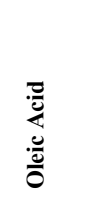 & 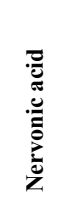 & 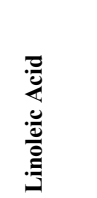 & 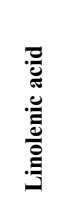 & 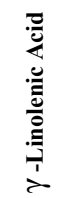 \\
\hline AK-4 & $13.92 \mathrm{a}$ & $36.98 \mathrm{~g}-\mathrm{w}$ & $31.82 \mathrm{a}-1$ & $0.94 \mathrm{~b}-1$ & $25.19 b-q$ & $2.59 \mathrm{a}-\mathrm{e}$ & $0.60 \mathrm{c}-\mathrm{h}$ & $22.40 \mathrm{~g}-\mathrm{n}$ & $1.03 \mathrm{~b}-1$ & $44.46 a-z$ & $0.12 b$ & $0.30 \mathrm{c}-\mathrm{e}$ \\
\hline Delcerro & $13.52 \mathrm{a}-\mathrm{b}$ & $37.58 \mathrm{e}-\mathrm{w}$ & $33.89 \mathrm{a}-\mathrm{h}$ & $0.90 \mathrm{~b}-1$ & $26.50 \mathrm{a}-\mathrm{k}$ & $2.49 \mathrm{a}-\mathrm{e}$ & $0.64 c-h$ & $17.44 \mathrm{~m}-\mathrm{s}$ & $1.09 \mathrm{~b}-\mathrm{k}$ & $44.55 \mathrm{a}-\mathrm{z}$ & $0.07 \mathrm{~b}$ & $0.26 \mathrm{c}-\mathrm{e}$ \\
\hline YB 195 & $12.64 \mathrm{a}-\mathrm{e}$ & $43.08 \mathrm{a}-\mathrm{b}$ & $28.85 \mathrm{c}-\mathrm{j}$ & $0.81 \mathrm{c}-\mathrm{m}$ & $24.21 \mathrm{~g}-\mathrm{r}$ & 2.76a-d & $1.07 \mathrm{a}-\mathrm{b}$ & $29.15 b$ & $0.88 \mathrm{~d}-1$ & $39.07 a-b$ & $0.12 b$ & $0.47 \mathrm{~b}-\mathrm{c}$ \\
\hline Acala Maxa & $13.50 \mathrm{a}-\mathrm{c}$ & $39.91 \mathrm{~b}-\mathrm{m}$ & $27.33 e-j$ & $1.04 \mathrm{~b}-\mathrm{e}$ & $27.82 \mathrm{a}-\mathrm{b}$ & $2.37 \mathrm{~b}-\mathrm{e}$ & $0.69 \mathrm{c}-\mathrm{h}$ & $19.89 \mathrm{a}-1$ & $0.96 \mathrm{~b}-1$ & $46.85 \mathrm{~d}-\mathrm{v}$ & $0.04 \mathrm{~b}$ & $0.12 \mathrm{e}$ \\
\hline YB 196 & $12.61 \mathrm{a}-\mathrm{f}$ & 40,70b-h & $29.58 b-j$ & $1.06 \mathrm{~b}-\mathrm{d}$ & $27.58 \mathrm{a}-\mathrm{d}$ & 2.60a-d & $0.66 \mathrm{c}-\mathrm{h}$ & $28.17 \mathrm{c}$ & $1.09 \mathrm{~b}-\mathrm{k}$ & $37.84 \mathrm{~b}$ & $0.08 \mathrm{~b}$ & $0.38 b-d$ \\
\hline Veramine & $11.79 \mathrm{a}-\mathrm{h}$ & $39,92 \mathrm{~b}-\mathrm{m}$ & $28.36 \mathrm{~d}-\mathrm{j}$ & $1.02 \mathrm{~b}-\mathrm{g}$ & $26.72 \mathrm{a}-\mathrm{k}$ & $2.62 \mathrm{a}-\mathrm{d}$ & $0.61 \mathrm{c}-\mathrm{h}$ & $24.69 \mathrm{c}-\mathrm{e}$ & $0.91 \mathrm{c}-1$ & $42.71 \mathrm{a}-\mathrm{z}$ & $0.07 \mathrm{~b}$ & $0.36 \mathrm{~b}-\mathrm{d}$ \\
\hline Acala 172 & $11.34 \mathrm{a}-\mathrm{k}$ & $39,62 b-n$ & $33,49 a-1$ & $0.82 b-1$ & $24.90 \mathrm{~b}-\mathrm{q}$ & $2.69 \mathrm{a}-\mathrm{d}$ & $0.57 \mathrm{e}-\mathrm{h}$ & $20.36 a-d$ & $0.97 \mathrm{~b}-1$ & $47.68 \mathrm{c}-\mathrm{v}$ & $0.28 b$ & $0.12 \mathrm{e}$ \\
\hline NGF 63 & $11.30 \mathrm{a}-\mathrm{k}$ & $39,42 b-o$ & $31.06 \mathrm{a}-1$ & $0.77 \mathrm{~d}-\mathrm{m}$ & $25.82 \mathrm{~b}-\mathrm{m}$ & $2.41 \mathrm{~b}-\mathrm{e}$ & $0.54 \mathrm{~g}-\mathrm{h}$ & $14.39 \mathrm{u}$ & $1.17 \mathrm{~b}-\mathrm{h}$ & $54.2 \mathrm{a}$ & $0.13 b$ & $0.31 \mathrm{c}-\mathrm{e}$ \\
\hline 1118-Glandless & $11.15 \mathrm{a}-\mathrm{k}$ & $38.22 \mathrm{~d}-\mathrm{u}$ & $31.37 \mathrm{a}-1$ & $1.15 \mathrm{~b}$ & $29.37 \mathrm{a}$ & $2.26 \mathrm{~b}-\mathrm{f}$ & $0.67 \mathrm{c}-\mathrm{h}$ & $20.03 a-1$ & $0.94 \mathrm{~b}-1$ & $45.37 \mathrm{k}-\mathrm{y}$ & $0.12 b$ & $0.35 b-d$ \\
\hline Paymaster 2379 & $10.79 a-1$ & $34.16 \mathrm{w}$ & $35.13 \mathrm{a}-\mathrm{d}$ & $1.04 \mathrm{~b}-\mathrm{e}$ & $25.12 b-q$ & $2.18 \mathrm{~b}-\mathrm{f}$ & $0.62 \mathrm{c}-\mathrm{h}$ & $22.49 \mathrm{~g}-\mathrm{q}$ & $0.95 \mathrm{~b}-1$ & $45.06 \mathrm{~m}-\mathrm{z}$ & $0.15 b$ & $0.34 \mathrm{~b}-\mathrm{d}$ \\
\hline Gossipolsüz 86 & $10.27 \mathrm{~d}-1$ & 36.591-w & $30.62 a-1$ & $0.90 \mathrm{~b}-1$ & $26.70 \mathrm{a}-\mathrm{k}$ & $2.5 \mathrm{a}-\mathrm{e}$ & $0.60 \mathrm{~d}-\mathrm{h}$ & $17.30 \mathrm{n}-\mathrm{s}$ & $0.99 b-1$ & $50.61 \mathrm{a}-1$ & $0.06 \mathrm{~b}$ & $0.30 \mathrm{c}-\mathrm{e}$ \\
\hline $152-\mathrm{F}$ & $10.23 \mathrm{~d}-1$ & $39,86 \mathrm{~b}-\mathrm{m}$ & $30.42 a-j$ & $0.91 \mathrm{~b}-1$ & $26.59 \mathrm{a}-\mathrm{k}$ & $2.47 \mathrm{~b}-\mathrm{e}$ & $0.87 \mathrm{a}-\mathrm{f}$ & $21.46 \mathrm{~h}-\mathrm{t}$ & $1.0 \mathrm{~b}-1$ & $45.20 \mathrm{k}-\mathrm{z}$ & $0.11 \mathrm{~b}$ & $0.36 \mathrm{~b}-\mathrm{d}$ \\
\hline Cirpan 60 & $10.15 \mathrm{~d}-1$ & $34.44 \mathrm{v}-\mathrm{w}$ & $37.7 \mathrm{a}$ & $0.67 \mathrm{i}-\mathrm{m}$ & $23.78 \mathrm{k}-\mathrm{s}$ & $2.67 \mathrm{a}-\mathrm{d}$ & $0.67 \mathrm{c}-\mathrm{h}$ & $20.52 a-c$ & $1.08 \mathrm{~b}-\mathrm{k}$ & $49.18 \mathrm{a}-\mathrm{t}$ & $0.14 \mathrm{~b}$ & $0.34 c-d$ \\
\hline Cukurova 1518 & $9.92 \mathrm{~d}-1$ & $39.31 \mathrm{~b}-\mathrm{o}$ & $30.14 b-j$ & $0.71 \mathrm{e}-\mathrm{m}$ & $22.91 \mathrm{~m}-\mathrm{s}$ & $2.40 \mathrm{~b}-\mathrm{e}$ & $0.64 \mathrm{c}-\mathrm{h}$ & 19.43a-1 & $1.19 \mathrm{~b}-\mathrm{e}$ & $50.42 \mathrm{a}-\mathrm{m}$ & $0.09 b$ & $0.30 \mathrm{c}-\mathrm{e}$ \\
\hline Albania 6172 & $9.67 \mathrm{~d}-1$ & $41.93 \mathrm{~b}-\mathrm{c}$ & $33.49 \mathrm{a}-1$ & $1.14 \mathrm{~b}-\mathrm{c}$ & 27.61a-d & $2.62 \mathrm{a}-\mathrm{d}$ & $0.80 \mathrm{a}-\mathrm{f}$ & $24.77 \mathrm{c}-\mathrm{e}$ & $0.92 \mathrm{c}-1$ & $40.86 a-z$ & $0.15 b$ & $0.38 b-d$ \\
\hline Fibermax 958 & $8.96 \mathrm{~h}-1$ & $35.19 \mathrm{p}-\mathrm{w}$ & $32.28 \mathrm{a}-1$ & $0.72 \mathrm{e}-\mathrm{m}$ & $25.06 \mathrm{~b}-\mathrm{q}$ & $3.0 \mathrm{a}-\mathrm{b}$ & $0.44 \mathrm{~h}$ & 19.96a-1 & $0.90 \mathrm{~d}-1$ & $49.84 a-q$ & $0.26 \mathrm{~b}$ & $0.31 \mathrm{c}-\mathrm{e}$ \\
\hline Garant & $9.65 \mathrm{~d}-1$ & $41.26 \mathrm{~b}-\mathrm{e}$ & $26.291-\mathrm{j}$ & $1.01 \mathrm{~b}-\mathrm{h}$ & $25.99 b-1$ & $2.58 \mathrm{a}-\mathrm{e}$ & $0.66 \mathrm{c}-\mathrm{h}$ & $20.83 \mathrm{~m}-\mathrm{z}$ & $0.95 \mathrm{~b}-1$ & $45.53 \mathrm{j}-\mathrm{y}$ & $0.09 \mathrm{~b}$ & $0.35 b-d$ \\
\hline Nazilli M-503 & $9.24 \mathrm{~h}-1$ & $40.40 \mathrm{~b}-\mathrm{j}$ & $28.44 \mathrm{~d}-\mathrm{j}$ & $0.83 b-1$ & $22.17 \mathrm{q}-\mathrm{s}$ & $2.13 b-f$ & $0.60 \mathrm{~d}-\mathrm{h}$ & $16.78 \mathrm{p}-\mathrm{q}$ & $1.02 \mathrm{~b}-1$ & $41.02 \mathrm{a}-\mathrm{z}$ & $0.09 b$ & $0.31 \mathrm{c}-\mathrm{e}$ \\
\hline Stoneville 8a & $9.06 \mathrm{~h}-1$ & $39,96 b-1$ & $31.49 \mathrm{a}-1$ & $0.76 \mathrm{~d}-\mathrm{m}$ & $20.97 \mathrm{~s}$ & $2.62 \mathrm{a}-\mathrm{d}$ & $0.63 \mathrm{c}-\mathrm{h}$ & $22.38 \mathrm{~g}-\mathrm{n}$ & $1.09 \mathrm{~b}-\mathrm{k}$ & $45.62 j-y$ & $0.12 b$ & $0.30 \mathrm{c}-\mathrm{e}$ \\
\hline Stoneville 468 & $8,99 \mathrm{~h}-1$ & $37.98 \mathrm{~d}-\mathrm{w}$ & $31.93 a-1$ & $1.59 \mathrm{a}$ & 26.51a-k & $1.60 \mathrm{~b}-\mathrm{f}$ & $1.22 \mathrm{a}$ & $33.15 \mathrm{a}$ & $2.71 \mathrm{a}$ & $52.52 \mathrm{a}-\mathrm{c}$ & $0.27 b$ & $0.30 \mathrm{c}-\mathrm{e}$ \\
\hline Fantom & $8.39 \mathrm{j}-1$ & $46.33 \mathrm{a}$ & $23.11 \mathrm{j}$ & $0.82 \mathrm{~b}-1$ & $25.52 b-p$ & $2.73 \mathrm{a}-\mathrm{d}$ & $0.56 \mathrm{~g}-\mathrm{h}$ & $17.68 \mathrm{k}-\mathrm{s}$ & $1.31 \mathrm{~b}$ & 51.13a-i & $0.07 \mathrm{~b}$ & $0.34 \mathrm{c}-\mathrm{d}$ \\
\hline Maydos Yerlisi & $8.39 \mathrm{j}-1$ & 37.31e-w & $32.43 a-1$ & $0.48 \mathrm{~m}$ & $24.26 \mathrm{f}-\mathrm{r}$ & $3.97 \mathrm{a}$ & $0.95 \mathrm{a}-\mathrm{d}$ & $18.36 \mathrm{e}-\mathrm{q}$ & $1.11 \mathrm{~b}-\mathrm{j}$ & $48.70 \mathrm{~b}-\mathrm{t}$ & $0.05 \mathrm{~b}$ & $0.41 b-d$ \\
\hline Okra Frego & 7.911 & $36.82 \mathrm{~h}-\mathrm{w}$ & $30.28 b-j$ & $0.88 \mathrm{~b}-1$ & 26.91a-1 & $2.36 \mathrm{~b}-\mathrm{e}$ & $0.63 \mathrm{c}-\mathrm{h}$ & $18.64 b-p$ & $1.04 \mathrm{~b}-1$ & $47.98 \mathrm{c}-\mathrm{v}$ & $0.17 \mathrm{~b}$ & $0.31 \mathrm{c}-\mathrm{e}$ \\
\hline Askabat 100 & $10.27 \mathrm{~d}-1$ & $39.5 b-n$ & $30.62 a-1$ & $0.89 \mathrm{~b}-1$ & $26.24 \mathrm{~b}-\mathrm{k}$ & $2.50 \mathrm{a}-\mathrm{e}$ & $0.66 \mathrm{c}-\mathrm{h}$ & 19.98a-1 & $1.12 \mathrm{~b}-\mathrm{i}$ & $47.49 \mathrm{c}-\mathrm{v}$ & $0.11 \mathrm{~b}$ & $0.30 \mathrm{c}-\mathrm{e}$ \\
\hline Sayar 314 & $10.42 \mathrm{~b}-1$ & $37.04 \mathrm{~g}-\mathrm{w}$ & $30.55 \mathrm{a}-1$ & $0.96 \mathrm{~b}-\mathrm{k}$ & $27.67 \mathrm{~A}-\mathrm{d}$ & $0.81 \mathrm{f}$ & $0.62 \mathrm{c}-\mathrm{h}$ & $20.68 \mathrm{a}-\mathrm{z}$ & $1.01 \mathrm{~b}-1$ & $47.03 \mathrm{c}-\mathrm{v}$ & $0.18 \mathrm{~b}$ & $0.33 \mathrm{c}-\mathrm{e}$ \\
\hline Bahar 14 & $11.13 \mathrm{a}-\mathrm{k}$ & $36.29 \mathrm{k}-\mathrm{w}$ & $0.96 \mathrm{~b}-\mathrm{k}$ & $0.78 \mathrm{~d}-\mathrm{m}$ & $24.76 \mathrm{c}-\mathrm{q}$ & $2.9 \mathrm{a}-\mathrm{b}$ & $0.80 \mathrm{~b}-\mathrm{f}$ & $24.35 c-f$ & $0.72 b-1$ & $44.32 \mathrm{a}-\mathrm{z}$ & $0.71 \mathrm{a}$ & $0.34 \mathrm{c}-\mathrm{d}$ \\
\hline BSC-4 & 11.17a-k & $39.62 b-n$ & $0.621-\mathrm{m}$ & $0.621-\mathrm{m}$ & $24.18 \mathrm{~g}-\mathrm{r}$ & 2.6a-d & $0.69 \mathrm{c}-\mathrm{h}$ & $23.22 \mathrm{e}-\mathrm{h}$ & 0.711 & $43.97 \mathrm{a}-\mathrm{z}$ & $0.07 \mathrm{~b}$ & $0.42 b-d$ \\
\hline Suregrow 125 & $9.37 \mathrm{~g}-1$ & $38.04 \mathrm{~g}-\mathrm{w}$ & $31.41 \mathrm{a}-1$ & $0.87 \mathrm{~b}-1$ & $24.36 \mathrm{e}-\mathrm{q}$ & $2.16 \mathrm{~b}-\mathrm{f}$ & $0.6 \mathrm{~d}-\mathrm{h}$ & $17.15 \mathrm{o}-\mathrm{s}$ & $0.89 \mathrm{~d}-1$ & $46.60 \mathrm{~d}-\mathrm{v}$ & $0.18 \mathrm{~b}$ & $0.69 \mathrm{a}$ \\
\hline GB 58 & $11.33 \mathrm{a}-\mathrm{k}$ & $39.35 \mathrm{~b}-\mathrm{o}$ & $28.50 \mathrm{~d}-\mathrm{j}$ & $0.82 \mathrm{~b}-\mathrm{m}$ & $24.36 \mathrm{e}-\mathrm{q}$ & $2.73 \mathrm{a}-\mathrm{d}$ & $0.72 \mathrm{~b}-\mathrm{h}$ & $25.84 \mathrm{c}$ & $1.01 \mathrm{~b}-1$ & $39.74 a-z$ & $0.07 \mathrm{~b}$ & $0.37 \mathrm{~b}-\mathrm{d}$ \\
\hline Okra 201 & $10.88 \mathrm{a}-1$ & $38.54 \mathrm{c}-\mathrm{t}$ & $31.65 \mathrm{a}-1$ & $0.98 \mathrm{~b}-\mathrm{j}$ & $26.67 \mathrm{a}-\mathrm{k}$ & $3.0 \mathrm{a}-\mathrm{b}$ & $0.69 \mathrm{c}-\mathrm{h}$ & $24.60 c-e$ & $0.80 \mathrm{~h}-1$ & $40.79 a-z$ & $0.28 \mathrm{~b}$ & $0.36 \mathrm{~b}-\mathrm{d}$ \\
\hline
\end{tabular}

Note. Same letters in the columns are not statistically significant. 

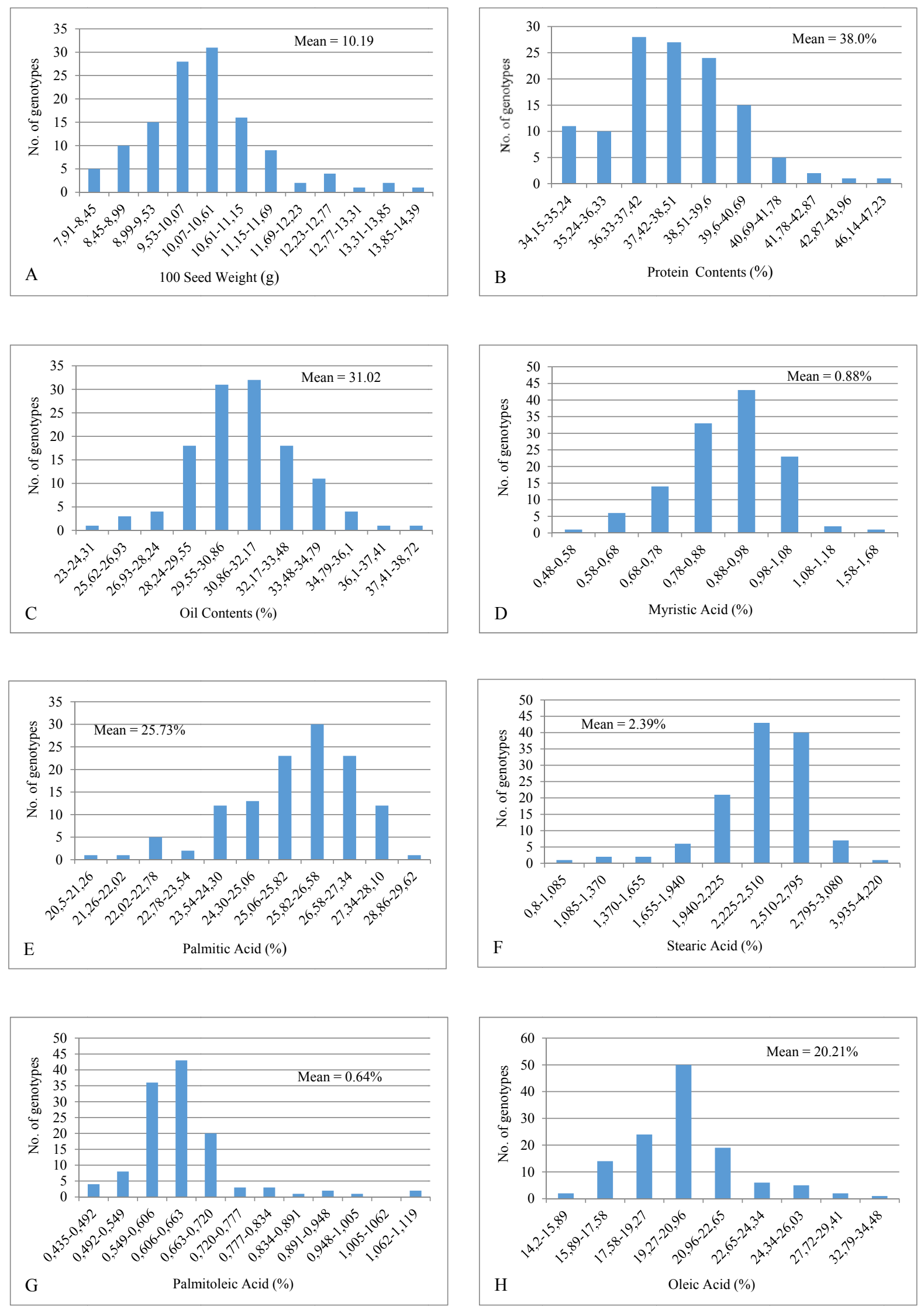

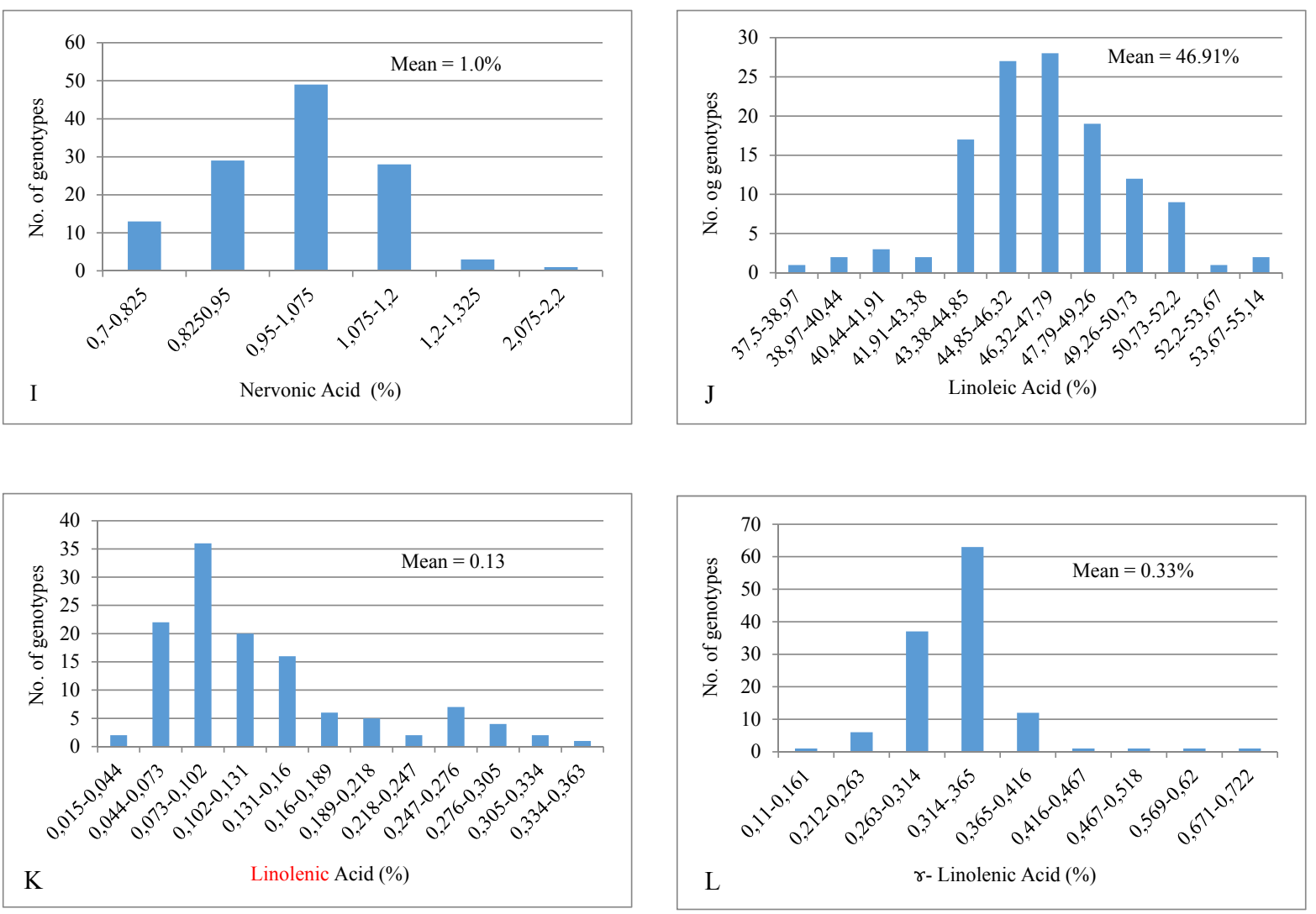

Figure 3. Frequency distribution of genotypes: A) 100 seed weight; B) Protein contents; C) Oil contents; D) Myristic acid; E) Palmitic acid; F) Stearic acid; G) Palmitoleic acid; H) Oleic acid; I) Nervonic acid; J) Linoleic acid; K) Linolenic acid; L) $\gamma$-Linolenic acid

The free fatty acids (FFA), which reflect the extent of chemical hydrolytic products in oil, were varied among the genotypes. Saturated fatty acids contain only single carbon to five carbon bonds and are chemically the least reactive. The saturated fatty acids have higher melting point than corresponding fatty acids of the same chain length with one or more double bonds (unsaturated fatty acids). Natural saturated fatty acids mostly have an unbranched structure with an even number of carbon atoms. Palmitic and stearic acids collectively constitute the saturated fatty acid. In earlier studies, the cotton genotypes and their hybrids were analyzed for fatty acid composition and significant variation was observed in their mean values ranging from 23.2 to $45.3 \%$ (Yunusova et al., 1991). Hall (2003) evaluated eleven cotton genotypes and reported greater genetic variability among the genotypes for fatty acids composition (ranged from 30.30 to $24.80 \%$ ) and that range could be used to screen the cotton germplasm for various environments. Dowd et al. (2010) studied fatty acid profile in 20 cotton genotypes at two different locations, and recorded significant differences among cotton genotypes for saturated fatty acids. O'Brien et al. (2005a, 2005b) findings revealed that cottonseed oil contains enough saturated fatty acids (25 to $26 \%$ : palmitic $22 \%$, stearic $3 \%$, myristic $1 \%$ ) to make it a relatively stable vegetable oil without partial hydrogenation as well as enough unsaturates (oleic $22 \%$, linoleic $\sim 52 \%$, and linolenic usually $<1 \%$ ) to make it a heart healthy oil. Mean myristic acid (\%) contents among the genotypes were $0.88 \%$ (Table 1). Sekhar et al. (2011) found that the average myristic acid value is $0.8 \%$; our results are similar to previous studies. Genotypes having highest myristic acid contents include Stoneville 468, 1118-Glandless and Albania 6172 (1.59, 1.15, $1.14 \%)$ while the genotypes having the lowest contents include Maydos Yerlisi $(0.48 \%)$ and BSC-4 (0.62\%) (Table 2). Palmitic acid is the most common fatty acid found in plants, micro-organisms and animals. In present study, varied values for palmitic acid were observed in the accessions. The palmitic acid contents ranged from 20.97 to $29.37 \%$ and mean value was 25.72 . The genotypes having highest amount of plamitic acid include 1118-Glandless (29.37\%), Acala Maxa (27.82\%) and Acala Prema (27.81\%) while Stoneville-8a and Nazilli M503 (20.97 and 21.31\%) respectively are having the lowest contents of palmitic acid (Table 2). Palmitic acid with an average value of $25.73 \%$ was reported in upland cotton (Hall, 2003); Sharma et al. (2009). Hamza et al. 
(1988) studied fatty acid composition and protein pattern in Egyptian cotton and found average values of 23.0 to 25.5\% among cotton genotypes. Dowd et al. (2010) observed that the concentration of palmitic acid, a saturated fatty acid, is higher in cottonseed oil ( $24 \%)$ than in many other vegetable oils. Average stearic acid contents $2.38 \%$ was observed and varied from 0.81 to $3.97 \%$ (Table 1). The genotypes having highest amount of stearic acid include Maydos Yerlisi (3.97\%), Okra 201 and Fibermax 958 (3\%) while the lowest percentage of stearic acid was observed in Sayar 314 (0.81\%) (Table 2). Wan et al. (1995b) analyzed stearic acid percentage in cottonseed and observed mean value of 2.1\%. Shekar et al. (2011) reaveled that 2.20\% stearic acid is found in cottonseed and (FAO/WHO Food Standard, 1999) has reported that the stearic acid contents should be 2.1 to $3.3 \%$ in cottonseed. Our study also revealed the same observations. The average amount of palmitoleic acid between genotypes were found to be $0.64 \%$ while palmitoleic acid concentration among genotypes varied $0.44 \%$ to $1.22 \%$ (Table 1). Stoneville 468 (1.22\%), YB195 (1.07\%), and Maydos Yerlisi (0.95\%) had upper values of palmitoleic acid while Fibermax $958(0.44 \%)$ had lowest value. Wan et al. (1995b) evaluated the cottonseed for palmitoleic acid percentage and mean contents should be $0.7 \%$. Dowd et al. (2010) studied the oil contents and showed mean value of $0.6 \%$ for palmitoleic acid and (FAO/WHO Food Standard, 1999) has described that highest palmitoleic acid should be upto $1.2 \%$. Results of present study are also consistent to earlier observations.

The unsaturated fatty acids contain one or more carbon double bonds and chemically more reactive than saturated fatty acids and this activity increases as the number of double bonds increase. Cottonseed oil generally consists of $70 \%$ unsaturated fatty acids. These acids are liquid at room temperature but begin to solidify at low temperature. In comparison, the unsaturated fatty acids solidify at more low temperature than saturated fatty acids. Oleic and linoleic acid collectively make the unsaturated fatty acids. Lukonge et al. (2007) evaluated 24 upland cotton genotypes for fatty acid profile and noted significant differences among genotypes for unsaturated fatty acids ranging from 70.2 to $74.9 \%$. Lawhon et al. (1977) studied seed composition of eight each glanded and glandless cotton genotypes and observed varied values for unsaturated fatty acids (70.0 to 79.6\%). Oleic acid occurs as the esters, commonly the triglycerides, which are the greasy materials in cottonseed oil. The free fatty acids which reflect the extent of enzymatic or chemical hydrolytic products in oil were varied over 14.39 to $33.15 \%$ as oleic acid (Table 1). NGF 63 (14.39\%) having minimum value while Stoneville 468 (33.15\%), YB 195 (29.15\%) and YB 196 (28.17\%) has higher oleic acid contents (Table 2). These values are comparable with most varieties of the cotton seed oils investigated in the literature Sharma et al. (2009). We found mean oleic acid contents of $20.21 \%$ (Table 1) which are in accordance with the Dowd et al. (2010); Sekhar and Rao (2011). The average amount of nervonic acid among genotypes found $1 \%$ and it varies from 0.71 to $2.71 \%$ (Table 1 ). Stoneville $468(2.71 \%)$ and Fantom $(1.31 \%)$ are the genotypes producing the highest content of nervonic acid while BSC-4 (0.71\%) and Bahar $14(0.72 \%)$ are having minimum amount (Table 2). Nervonic acid is a new synthesised fatty acid which will be good addition to fatty acids. Mathaus and Ozcan (2015) observed linoleic and $\gamma$-linolenic acid contents in cottonseed 53.2\% while average amount of linoleic acid in our study among genotypes was $46.81 \%$ and varied from 37.84 to $54.20 \%$. The genotypes having highest linoleic acid concentration include NGF 63 (54.20\%), and Stoneville 468 (52.52\%) while least concentration of linoleic acid contents found in YB 196 (37.84\%), YB 195 (39.07\%) and GB 58 (39.74\%) (Table 2). These values are consistent to findings of Dowd et al. (2010); Sekhar and Rao (2011); Mathaus and Ozcan (2015). Linolenic acid contents varied from 0.02 to 0.71 while mean $\gamma$-linolenic acid contents $0.33 \%$ observed among genotypes (Table 1). $\gamma$-linolenic contents varied from 0.12 to $0.69 \%$ and genotypes having highest contents include Suregrow 125 (0.67\%), Paymaster 330 (0.57\%), YB 195 (0.47\%) and BSC-4 (0.42\%) while Acala Maxa (0.12\%) having lowest contents (Table 2). In our study, it is considered that this additional fatty acid ( $\gamma$-linolenic) will serve a milestone for developing data base. With the differences between genotypes, it can be inferred that there is genetic variation among the genetic stock, demonstrating the possibilities of obtaining genetic improvement by applying the selection.

\subsection{Principal Component Analysis \& Correlation for Determining Genotypic Relationship for Different Traits}

The principal component analysis (Figure 1) and correlation analysis (Table 3) were conducted among genotypes to identify relationships for observed traits. Principal component analysis shown in the Figure 1 which reveals significant differences between genotypes for all traits. It is assumed a complex opposite relationship with a total protein content and oil (The same way arrows shows positive relation among each other and the reverse shows a negative relationship). The amount of linoleic acid appears to be associated with other characters in the opposite direction through analysis, on the other hand nervonic acid is having positive relationship with palmitic acid and myristic acid. Myristic acid and palmitic acid that are affected each other positively when traits are compared together, myristic acid and linoleic acid affect each other negatively (open arrows are the reduction properties that affect relation each other in positive direction, if the angle is increased the traits are affected negatively). 
The results are similar to correlation analysis as shown in Table 3. Linoleic acid and oil contents are negatively correlated to protein, palmitoleic acid, oleic, stearic and $\gamma$-linolenic acid (Figure 1). Genotypes are shown by numbers in Figure 2. Siginificant variation observed among genotypes by principal component analysis. The statistical analysis showed that highest contents of palmitoleic acid, myristic acid, oleic acid, linoleic and nervonic acid observed in Stoneville 468 followed by YB 195 having highest protein contents. YB196 also contain high amount of oleic acid. 1118 Glandless and Albania 6172 had higher amount of myristic acid while Bahar 14, Bahar 82 and 4-SB had higher amounts of linolenic acid. Moreover, Suregrow 125, Paymaster 330 and YB 195 had higher amounts of $\gamma$-linolenic acid while Maydos Yerlisi, Okra 201and Fibermax 958 had higher amounts of stearic acid contents.

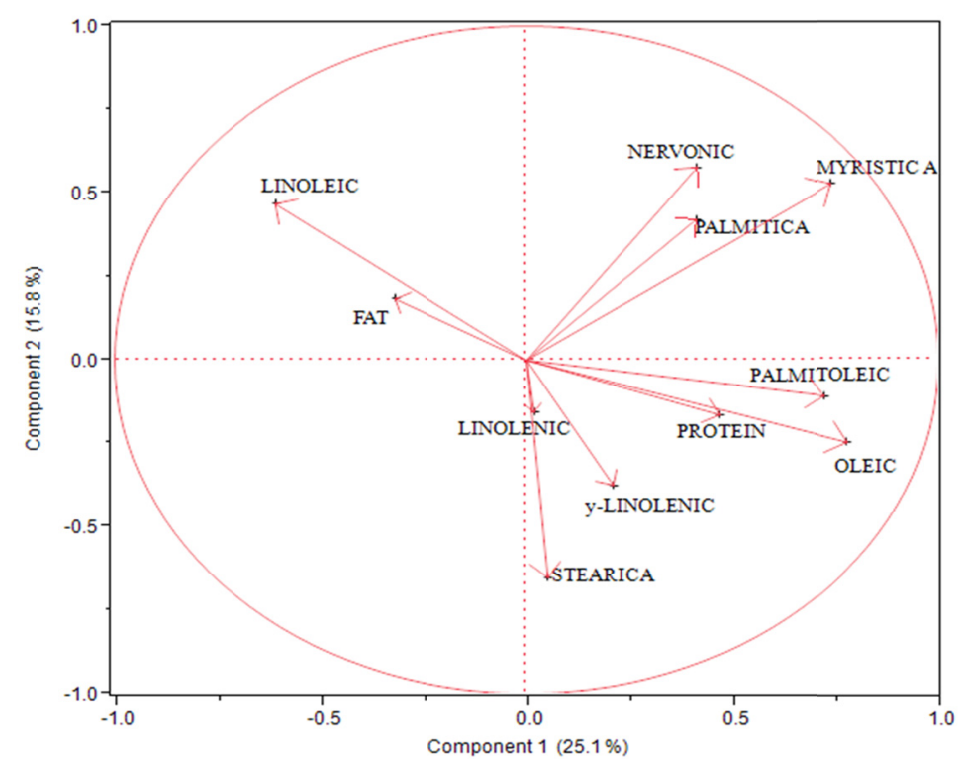

Figure 1. Principal component analysis for the relationship of traits

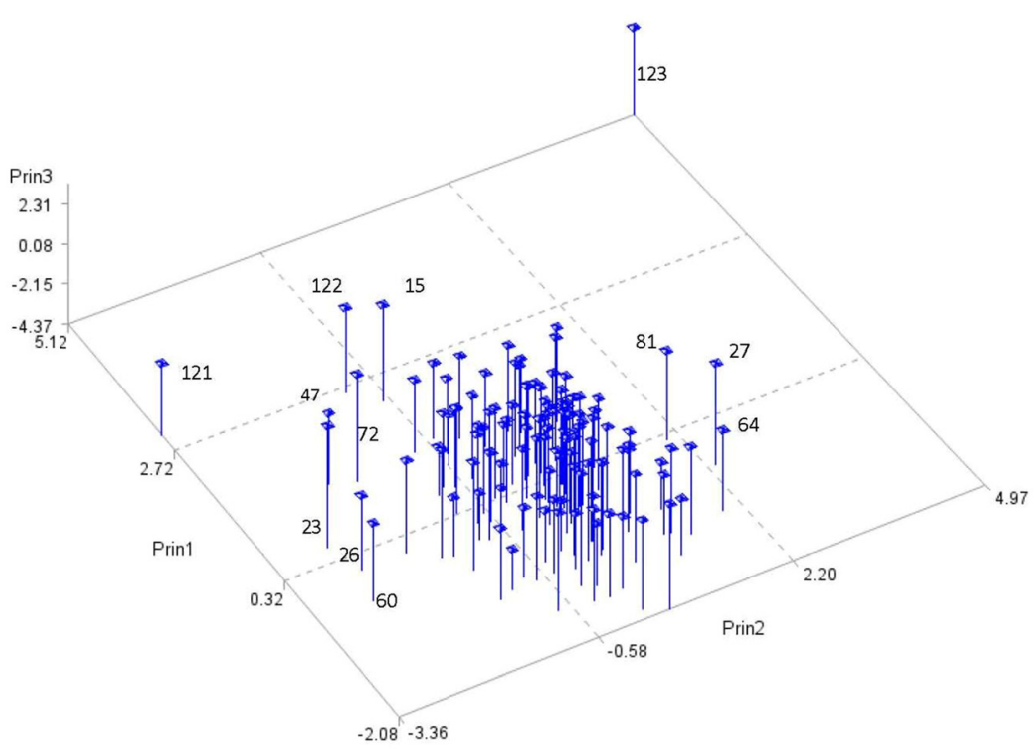

Figure 2. Principal component analysis for the relationship among genotypes $(15=$ Albania $6172,23=$ Bahar 14 , $26=$ BSC-4, $27=$ Ca-228, $47=$ GB 58, $60=$ Maydos Yerlisi, $64=$ Nata, $72=$ Okra 201, 81 $=$ Sayar 314, $121=$ YB $195,122=$ YB 196, $123=$ Stoneville 468) 
Table 3. Pearson correlation coefficients to examine the relationship of traits

\begin{tabular}{|c|c|c|c|c|c|c|c|c|c|c|c|}
\hline & $\begin{array}{l}100 \text { Seed } \\
\text { weight }\end{array}$ & $\begin{array}{l}\text { Myristic } \\
\text { acid }\end{array}$ & $\begin{array}{l}\text { Palmitic } \\
\text { acid }\end{array}$ & $\begin{array}{l}\text { Palmitoleic } \\
\text { acid }\end{array}$ & $\begin{array}{l}\text { Stearic } \\
\text { acid }\end{array}$ & $\begin{array}{l}\text { Oleic } \\
\text { acid }\end{array}$ & $\begin{array}{l}\text { Linoleic } \\
\text { acid }\end{array}$ & $\gamma$-Linolenic & $\begin{array}{l}\text { Linolenic } \\
\text { acid }\end{array}$ & $\begin{array}{l}\text { Nervonic } \\
\text { acid }\end{array}$ & Protein \\
\hline \multicolumn{12}{|l|}{100 seed weight } \\
\hline Myristic acid & 0.121 & & & & & & & & & & \\
\hline Palmitic acid & 0.177 & $0.637 * *$ & & & & & & & & & \\
\hline Palmitoleic acid & 0.170 & $0.276^{* *}$ & 0.048 & & & & & & & & \\
\hline Stearic acid & -0.003 & -0.149 & -0.037 & 0.100 & & & & & & & \\
\hline Oleic acid & $0.255^{* *}$ & $0.339 * *$ & 0.067 & $0.526^{* *}$ & 0.102 & & & & & & \\
\hline Linoleic acid & $-0.345^{* *}$ & $-0.251 * *$ & $-0.175^{* *}$ & $-0.241 * *$ & $-0.161^{*}$ & $-0.527 * *$ & & & & & \\
\hline$\gamma$-Linolenic & -0.043 & 0.020 & 0.066 & 0.086 & 0.062 & 0.131 & $-0.224 * *$ & & & & \\
\hline Linolenic & 0.012 & 0.005 & -0.019 & 0.075 & 0.134 & 0.178 & 0.056 & -0.060 & & & \\
\hline Nervonic & $-0.168 * *$ & $0.381 * *$ & 0.071 & $0.322 * *$ & $-0.174 * *$ & $0.241 * *$ & $0.181 * *$ & -0.048 & -0.094 & & \\
\hline Protein & $0.150^{*}$ & $0.177^{* *}$ & 0.047 & $0.180^{* *}$ & 0.060 & $0.198^{*}$ & $-0.180 * *$ & 0.052 & $-0.200 * *$ & 0.083 & \\
\hline Oil & $-0.143 *$ & -0.077 & 0.053 & $-0.153 *$ & -0.019 & -0.090 & 0.101 & 0.093 & -0.002 & -0.0006 & $-0.420 * *$ \\
\hline
\end{tabular}

Note. ${ }^{* *}$ : Highly significant correlation $(\mathrm{p}<0.01)$; : Significant correlation $(\mathrm{p}<0.05)$.

Based on these associations, one can speculate on what cottonseed oil modifications might be possible among cotton genotypes. Lower levels of palmitic acid, linoleic acid and higher levels of oleic acid and possibly stearic acid would be ideal (Dowd et al., 2010). Based on the correlations, however, this ideal distribution appears unlikely. Hence, it appears unlikely both acids can be simultaneously reduced. Higher levels of oleic acid were correlated with lower levels of linoleic acid, so this change seems likely, but possibly with a concomitant increase in the level of myristic acid. Hence, a reduction of myristic acid and an increase in oleic acid also does not appear favorable. Because cyclopropenoid fatty acids did not correlate strongly with any other fatty acid components, it may be possible to reduce their concentration without negatively influencing other compositional factors. Association among oil, protein and fatty acid contents among genotypes examined through correlation and principal component analysis (Figure 1 and Table 3). This study also detected significant negative correlation between oil and protein content in cottonseed, as Turner et al. (1976b); Leffler et al. (1977); Hanny et al. (1978); Shaver and Dilday (1982) reported. Most of the observed correlations appeared to have some foundation within fatty acid biosynthesis pathways. Significant positive correlations were generally observed between neighboring fatty acids when the two acids were removed from pathway branch points. Tunc and Duman (2007) used gravimetric methods for fuzzy seeds and delinted seed for the determination of protein contents and found 20.7, 23.0 and $35.7 \%$, respectively. Results shows positive correlation $(\mathrm{p}<0.01)$ between 100 seed weight and oleic acid, significant positive correlation $(\mathrm{p}<0.05)$ between 100 seed weight and protein content and non-significant correlation between 100 seed weight and linolenic acid, myristic acid, palmitoleic and palmitic acid (Table 3). Non-significant positive correlation $(\mathrm{p}<0.05)$ observed between protein and oil contents. For example positive correlation observed among important traits myristic acid-palmitic acid, palmitoleic acid, oleic acid, nervonic acid and myristic acid-protein content. A similar trend was apparent $(\mathrm{p}<0.05)$ among linoleic acid and nervonic acid while non-significant correlation among stearic acid-oleic acid, linolenic acid, $\gamma$-linolenic acid and protein contents. Moreover, significant positive relation present among traits including palmitoleic acid compared to oleic acid, nervonic acid and protein content; while non-significant correlation was between oleic acid and linolenic acid and $\gamma$-linolenic acid while significant negative correlation $(\mathrm{p}<0.05)$ found between palmitoleic acid and oil content and linoleic acid. Significant negative correlation observed between palmitoleic acid and linoleic acid, while non-significant correlation was between palmitoleic acid and $\gamma$-linolenic and stearic acid contents. We used standard FAME 37 for determining the fatty acid contents in oil as it is having higher amount of fatty acids. The synthesis of nervonic acid will be good addition in fatty acids.

\section{Discussions}

The relatively high level of palmitic acid naturally present in cottonseed oil has been an important contributor to the stability of the oil and to the solidity of its hydrogenated derivatives, but it is nutritionally undesirable. Because the increases in oleic acid or stearic acid are able to impart the required functional properties on the 
modified oils, it should now be possible to dramatically lower palmitic acid in cottonseed oil without compromising performance (Liu et al., 2002). Lawhon et al. (1977) studied seed composition of eight glanded and eight glandless cotton genotypes produced at various locations. Only limited variation in fatty acid composition was reported and the glandless trait did not significantly affect seed oil composition. Hamza et al. (1988); Nergiz et al. (1997); Lukonge et al. (2007) studied fatty acid profiles of small numbers of genotypes). As with the Lawhon et al. (1977) study, these studies showed fairly limited variation in fatty acid composition.

Based on these associations, one can speculate on what cottonseed oil modifications might be possible among agronomic cotton genotypes. Correlation analysis does not establish cause and effect, and these inferences are based on a small number of cotton genotypes representing a narrow range of cotton genetics. In addition, genetically modified cottonseeds have been reported with compositions that do not support the correlations identified in the current study. Specifically, Liu et al. (2002) mentioned that down-regulated FAD-II in cotton resulted in reduced levels of both palmitic acid and linoleic acid. This difference emphasizes the complicated associations that likely exist among oilseed fatty acids, which can be influenced by processes that include not only acid synthesis but also transfer of acids between various carrier molecules and triglycerides. Correlation analysis revealed significant positive correlation between the amount of protein and 100 seed weight and negative correlation was observed for oil contents while highly significant neagtive relation was observed among protein and oil contents. Bellaloui and Turley (2013), observed the relationship among protein and oil contents under greenhouse conditions, and found that fuzzless cotton seeds accumulated higher oil and lower protein content compared with their equivalent fuzzy seeds, showing an inverse relationship between protein and oil contents. The inverse relationship between amount of protein and oil was previously reported in other species such as corn (Kebede et al., 2013), soybean (Burton, 1985; Ray et al., 2006; Bellaloui et al., 2013a, 2013b), and cottonseed (Pettigrew \& Dowd, 2011). Only a few studies have attempted to discuss variability in cottonseed fatty acid profiles, the source of the variation, or how fatty acid percentages correlate with each other. Lawhon et al. (1977) studied seed composition of eight each glanded and glandless cotton genotypes and observed varied values for unsaturated fatty acids (70.0 to 79.6\%). Hamza et al. (1988); Nergiz et al. (1997); Lukonge et al. (2007) studied fatty acid profiles of small numbers of genotypes and observed negative correlation palmitic acid and linoleic acid contents grown at a single location for a single year.

Statistically, fatty acid contents showed differences for all traits except myristic acid and linolenic acid. The range of measured variation in the individual fatty acids in this work was similar to the range of variation reported in most prior studies (Yunusova et al., 1991). The range of values for individual fatty acids tended to be slightly broader than the range reflected in the Codex alimentarius trading standard for cottonseed oil (FAO/WHO Food Standards, 1999). Although genetics accounted for a significant amount of fatty acid variation, the overall range of variation was insufficient to indicate that breeding within this population would produce desirable changes in oil composition. Efforts to breed cotton plants for improved oil properties will require a broader survey of cotton germplasm, perhaps including other Gossypium species, or possibly mutagenesis-based developmental efforts, which have been useful for modifying fatty acid profiles of other oilseeds (Fehr et al., 1991; Osorio et al., 1995). Vegetable oils like cotton seed oil are typically rich in C 18-poly unsaturated fatty acids mainly linoleic acid and $\gamma$-linolenic acid. These fatty acids are essential fatty acids because they are precursors of n-3 and n-6 long chain poly unsaturated fatty acids such as eicosapentaenoic acid, docosahexaenoic acid and arachidonic acid, and also human body can not synthesize them through de novo mechanism. Thus, linoleic acid and $\gamma$-linolenic acid should be provided in the diet.

Variance analysis, frequency analysis and principal component analysis revealed that Stoneville 468, YB 195, YB 196, Albania 6172, GB 58, Maydos Yerlisi and Okra 201 are having desirable cottonseed traits. Stoneville 468 is a model cultivar for improving oil contents in breeding programs as having good protein and oil contents. Okra 201 and gossypol free lines (YB 195, YB 196) can be used as for increasing oil and protein contents.

\section{Conclusion}

The results of the present study revealed that most of the characteristics of cottonseed oils tested from Kahramanmras Sutcu Imam University were quite identical with cotton seed oil, protein and fatty acid contents of different countries. Regardless of the fact that some nutritionally important physicochemical attributes, for example, fatty acids were considerably varied but most of the other characteristics of cotton seed oils were quite comparable among the varieties selected. The genotypes show some variation for protein, oil contents and in fatty acid composition, and this variation is associated with genetics. As genotypes were observed to affect seed oil composition, additional examination is required to get better genotypes. Finally, little documentation exits on the genetic association among oil and fatty acid composition with fiber traits, and other useful traits, all of which must be considered when tailoring the cotton plant for value-added seed properties. 


\section{Acknowledgements}

This project was supported by Kahramanmaras Sutcu Imam University, Scientific Research Projects Coordination Unit (No: 2013/3-16YLS).

\section{References}

Abdelmoez, W., Abdelfatah, R., Tayeb, A., \& Yoshida, H. (2011). Extraction Cottonseed Oil Using Subcritical Water Technology. AIChE Journal, 57(9). http://dx.doi.org/10.1002/aic.12454

Agarwal, D. K., Singh, H., Chavan, H., \& Kate, N. (2003). Variability pattern for various seed oil traits in diploid cotton (Gossypium arboreum). Indian Journal of Agricultural Science, 73(2), 116-117.

Anonymous. (2006). Tarladan Pazara Endüstriyel ve Stratejik Tarım Ürünleri, Türkiye ve Dünyadaki Yeri, Sorunlar ve Çözüm Önerileri. Tarım Satış Kooperatifleri Birlikleri Toplantısı Sunumları. Değerlendirmeler ve Sonuç Bildirgesi, Mayıs 25-28, 2006, 306, Nevşehir.

Anonymous. (2009). Cottonseed Oil Production, Consumption on the Rise. Cotton Grower (p. 12). ProQuest Agricultural Science Collection.

Ash, M., \& Dohlman, E. (2006). Oil crops situation and outlook yearbook. Electronic outlook report from the Economic Research Service. United States Department of Agriculture, Washington, USA.

Ashokkumar, K., \& Ravikesevan, R. (2011). Conventional and molecular Breeding Approaches for Oil and Seed Protein Content İmprovement in Cotton. International Research Journal of Plant Science, 2(2), 037-045.

Azhar, F. M., \& Ahmad, M. (2000). Inheritance pattern of cotton seed oil in diverse germplasm of G. hirsutum L. Pak. J. Biol. Sci., 3, 1250-1252. http://dx.doi.org/10.3923/pjbs.2000.1250.1252

Bellaloui, N., \& Turley, R. B. (2013). Effects of fuzzless cottonseed phenotype on ottonseed nutrient composition in near isogenic cotton (Gossypium hirsutum L.) mutant lines under well-watered and water stress conditions. Front. Plant Sci., 4, 516. http://dx.doi.org/10.3389/fpls.2013.00516

Bellaloui, N., Gillen, A. M., Mengistu, A., Kebede, H., Fisher, D. K., Smith, J. R., et al. (2013a). Responses of nitrogen metabolism and seed nutrition to drought stress in soybean genotypes differing in slow-wilting phenotype. Front. Plant Sci., 4, 498. http://dx.doi.org/10.3389/fpls.2013.00498

Bellaloui, N., Hu, Y., Mengistu, A., Kassem, M. A., \& Abel, C. A. (2013b). Effects of foliar boron application on seed composition, cell wall boron, and seed $\mathrm{d} 15 \mathrm{~N}$ and $\mathrm{d} 13 \mathrm{C}$ isotopes in water-stressed soybean plants. Front. Plant Sci., 4, 270. http://dx.doi.org/10.3389/fpls.2013.00270

Burton, J. W. (1985). Breeding soybean for improved protein quantity and quality. In R. Shibles (Ed.), World Soybean Research Conference III: Proceedings (pp. 361-367). Ames; Boulder: Westview Press.

Calhoun, D. S., \& Bowman, D. T. (1999). In C. W. Smith \& J. T. Cothren (Eds.), Cotton origin, history, technology and production (pp. 361-414). John Wiley and Sons, New York.

Cherry, J. P., Kohel, R. J., Jones, L. A., \& Powell, W. H. (1981). Cottonseed quality: factors affecting feed and food uses. In J. M. Brown (Ed.), Proceedings of the Beltwide Cotton Production Reseasrch Conference (pp. 266-283). National Cotton Council, Memphis, USA.

Chesterfield, M., Rogers, P. L., \& Adesina, A. A. (2013). Liquid-liquid phase equilibrium studies of organicaqueous medium during biodiesel synthesis. Chemical Engineering Science, 104, 540-548. http://dx.doi.org/10.1016/j.ces.2013.09.015

Cruz, C. D., \& Carneiro, P. C. S. (2003). Modelos biométricos aplicados ao melhoramento genético (Vol. 2, p. 585). UFV, Vic, OSA.

Dani, R. G. (1989). Heterosis and combining ability for oil content and other economic traits in upland cotton ( $G$. hirsutum). Ind. J. Genet., 209, 209-212.

Dani, R. G. (1990). Genetic research of cottonseed oil: a review. Cotton Fibres Tropical., 45, 71-75.

Dani, R. G. (1991). Analysis of combining ability for seed oil content in cotton (G. hirsutum). Acta Agron. Hungarica, 40, 123-127.

Dowd, M. K., Boykin, D. L., Meredith, W. R., Campbell, B. T., Bourland, F. M., Gannaway, J. R., ... Zhang, J. F. (2010). Fatty acid profiles of cottonseed genotypes from the national cotton variety trials. J Cotton Sci, 14, 64-73. 
FAO/WHO Food Standards. (1999). Standard for named vegetable oils (Standard \#210). Retrieved July 12, 2010, from http://www.codexalimentarius.net/web/standard_list.do?lang=en

Fehr, W. R., Welke, G. A., Hammond, E. G., Duvick, D. N., \& Cianzio, S. R. (1991). Inheritance of reduced palmitic acid content in seed oil of soybean. Crop Sci., 31, 88-89. http://dx.doi.org/10.2135/cropsci1991.0011183X003100010022x

Gotmare, V., Singh, P., Mayee, C. D., Deshpande, V., \& Bhagat, C. (2004). Genetic Variability for Seed Oil Content and Seed İndex in Some Wild Species and Perennial Races of Cotton. Plant Breeding, 123, $207-208$. http://dx.doi.org/10.1046/j.1439-0523.2003.00914.x

Gul, S., Khan, N. U., Batool, S., Baloch, M. J., Munir, M., Sajid, M., .. Kazmi, S. F. (2014). Genotype by environment interaction and association of morpho-yield variables in upland cotton. J. Animal \& Plant Sci., 24(1), 262-271.

Hall, A. J. (2003). Evaluation of fatty acid composition of cotton germplasm and association with cold tolerance (M.S. Thesis, Texas Tech University).

Hamilton, K. A., Pyla, P. D., Breeze, M., Oslon, T., Li., M., Robinson, E., et al. (2004). Bollgard II cotton: compositional analysis and feeding studies of cottonseed from insect-protected cotton (Gossypium hirsutum L.) producing the Cry $1 \mathrm{Ac}$ and Cry 2Ab2 proteins. J. Agric. Food Chem., 52, 6969-6976. http://dx.doi.org/10.1021/jf030727h

Hamza, M. A., Abdel-Aal, M. H., \& Khalil, M. M. (1988). Studies on Egyptian cottonseeds: Fatty acid composition and protein patterns. Nahrung, 32, 255-259. http://dx.doi.org/10.1002/food.19880320313

Hanna, G. M., Dąbrowsk, Ł., \& Jacek, N. (2001). Accelerated Solvent Extraction (ASE) in the Analysis of Environmental Solid Samples-Some Aspects of Theory and Practice. Critical Reviews in Analytical Chemistry, 31(3), 149-165. http://dx.doi.org/10.1080/20014091076712

Hanny, B. W., Meredith, W. R., Bailey, J. C., \& Harvey, A. J. (1978). Genetic relationships among chemical constituents in seeds, flower buds, terminals, and mature leaves of cotton. Crop Sci., 18, 1071-1074. http://dx.doi.org/10.2135/cropsci1978.0011183X001800060042x

He, Z., Shankle, M., Zhang, H., Way, T. R., Tewolde, H., \& Uchimiya, M. M. (2013). Mineral composition of cottonseed is affected by fertilization management practices. Agron. J., 105, 341-350. http://dx.doi.org/10.2134/agronj2012.0351

International Cotton Advisory Committee. (2015). Statement of the 74th Plenary Meeting: "From Farm to Fabric: The Many Faces of Cotton". ICAC.

Jiwen, Y., Shuxun, Y., Shuli, F., Meizhen, S., Honghong, Z., Xingli, L., \& Zhang, J. (2012). Mapping quantitative trait loci for cottonseed oil, protein and gossypol content in a Gossypium hirsutum $\times$ Gossypium barbadense backcross inbred line population. Euphytica, 187, 191-201. http://dx.doi.org/10.1007/s10681-012-0630-3

Karahan, E. (2007). Gossypium hirsutum L. Türüne Ait Erkenci (Paum-15) Genotipi, Orta Erkenci (Çukurova 1518) Ve Geçci (Deltaopal) Pamuk Çeşitlerinin Yağ Oranı Ve Yağ Asitlerinin Saptanması. Ç.Ü. Fen Bilimleri Enstitüsü Yüksek Lisans Tezi.

Kebede, H., Abbas, H. K., Fisher, D. K., \& Bellaloui, N. (2013). Relationship between aflatoxin contamination and physiological responses of corn plants under drought and heat stress. Toxins, 4, 1385-1403. http://dx.doi.org/10.3390/toxins4111385

Khan, N. U. (2013). Diallel analysis of cotton leaf curl virus (CLCuV) disease, earliness, yield and fiber traits under CLCuV infestation in upland cotton. Aust. J. Crop Sci., 7(12), 1955-1966. http://dx.doi.org/10.5424/sjar/20110902-166-10

Khan, N. U., \& Hassan, G. (2011). Genetic effects on morphological and yield traits in cotton (G. hirsutum L.). Span. J. Agric. Res., 9(2), 460-472.

Khan, N. U., Hassan, G., Marwat, K. B., Farhatullah, M. B., Kumbhar, A., Parveen, U., ... Soomro, Z. A. (2009a). Diallel analysis of some quantitative traits in G. hirsutum L. Pakistan J. Bot., 41(6), 3009-3022.

Khan, N. U., Hassan, G., Marwat, K. B., Kumbhar, M. B., Khan, I., Soomro, Z. A., ... Kahan, M. Z. (2009). Legacy study of cottonseed traits in upland cotton using Griffing's combining ability model. Pak. J. Bot., 41(1), 131-142. 
Khan, S. A., Khan N. U., Mohammad, F., Ahmad, M., Khan, I. A., Bibi, Z., \& Khan I. U. (2011). Combining ability analysis in intraspecific F1 diallel cross of upland cotton. Pakistan J. Bot., 43(3), 1719-1723.

Kohel, R. J. (1980). Genetic studies of seed oil in cotton. Crop Sci., 20, $784-787$. http://dx.doi.org/10.2135/cropsci1980.0011183X002000060026x

Kohel, R. J., Glueck, J., \& Rooney, L. W. (1985). Comparison of cotton germplasm collections for seed-protein content. Crop Sci., 25, 961-963. http://dx.doi.org/10.2135/cropsci1985.0011183X002500060015x

Lawhon, J. T., Cater, C. M., \& Mattil, K. F. (1977). Evaluation of the food potential of sixteen varieties of cottonseed. J. Am. Oil Chem. Soc., 54, 75-80. http://dx.doi.org/10.1007/BF02912394

Leffler, H. R., Elmore, C. D., \& Hesketh, J. D. (1977). Seasonal and fertility-related changes in cottonseed protein $\begin{array}{lllll}\text { quantity and } & \text { quality. } & \text { Crop } & \text { 953-956. }\end{array}$ http://dx.doi.org/10.2135/cropsci1977.0011183X001700060034x

Liu, Q., Singh, S. P., \& Green, A. G. (2002). High-stearic and high-oleic cottonseed oils produced by hairpin RNAmediated post-transcriptional gene silencing. Plant Physiol., 129, 1732-1743. http://dx.doi.org/10.1104/pp.001933

Lukonge, E., Labuschagne, M. T., \& Hugo, A. (2007). The evaluation of oil and fatty acid composition in seed of cotton accessions from various countries. J. Sci. Food Agric., 87, $340-347$. http://dx.doi.org/10.1002/jsfa.2731

Matthaus, B., \& Ozcan, M. M. (2015). Oil content, fatty acid composition and distribution of Vitamine-E-Active componds of some fruit seed oils. Antioxidants, 4, 124-133. http://dx.doi.org/10.3390/antiox4010124

Nagappa, H., \& Khadi, B. M. (2011). Studies on composition of oil and fatty acid in Bt and non Bt cotton (G. hirsutum L.). World Cotton Res. Conf. 5, India (pp. 568-571).

Nergiz, C., Yalcin, H., \& Yildiz, H. (1997). Some analytical characters of cottonseed varieties grown in Turkey. Grasasy Aceites, 48, 411-414. http://dx.doi.org/10.3989/gya.1997.v48.i6.813

Nyanzi, S. A., Carsternsen, B., \& Schwack, W. (2005). A comparative study of the fatty acid profiles of Passiflora seed oils from Uganda. J. Am. Oil Chem. Soc., 82(1), 41-44. http://dx.doi.org/10.1007/s11746-005-1040-2

O’Brien, R. D., Jones, L. A., \& King, C. C., Wakelyn, P. J., \& Wan, P. J. (2005a). Cottonseed Oil. In F. Shahidi (Ed.), Bailey's Industrial Oil \& Fat Products (6th ed., pp. 173-279). John Wiley \& Sons, Inc. Hoboken, NJ, USA.

O’Brien, R. D., Wakelyn, P. J., \& Wan, P. J. (2005b). Cottonseed Oil: Alternative to Trans Fatty Acid Containing Oils. Oil Mill Gazetteer, 111, 2-4.

Osorio, J., Martinez, J. F., Mancha, M., \& Garces, R. (1995). Mutant sunflowers with high concentrations of saturated fatty acids in the oil. Crop Sci., $35, \quad 739-742$. http://dx.doi.org/10.2135/cropsci1995.0011183X003500030016x

Pahlavni, M. H., Miri, A. A., \& Kazemi, G. (2008). Response of oil and protein content to seed size in cotton. Int J. Agric. Biol., 10, 643-647.

Pettigrew, W. T., \& Dowd, M. K. (2011). Varying planting dates or irrigation regimes alters cottonseed composition. Crop Sci., 51, 2155-2164. http://dx.doi.org/10.2135/cropsci2011.02.0085

Quampah, A., Huang, R., Wu, Z., Liu, G. J., Li, H. Y., Zhu, J. R., \& Shi, C. H. (2012). Estimation of Oil Content and Fatty Acid Composition in Cottonseed Kernel Powder Using Near Infrared Reflectance Spectroscopy. Journal American Oil Chemical Society, 89, 567-575. http://dx.doi.org/10.1007/s11746-011-1945-2

Ramachandran, S., Singh, S. K., Larroche, C., Soccol, C. R., \& Pandey, A. (2007). Oil cakes and their biotechnological applications-A review. Bioresour Technol, 98(10), 2000-2009. http://dx.doi.org/10.1016/ j.biortech.2006.08.002

Ray, J. D., Fritschi, F. B., \& Heatherly, L. G. (2006). Large application of fertilizer N at planting affects seed protein and oil concentrations in the early soybean production system. Field Crops Res., 99, 67-74. http://dx.doi.org/10.1016/j.fcr.2006. 03.006

SAS. (2002-2012). Statistical Analysis System. Cary, NC: SAS Institute Inc.

Saxena, K. D., Sharma, S. K., \& Sambi, S. S. (2011). Comparative Extraction of Cotoonseed Oil by n-Hexan and Ethanol. ARPN Journal of Engineering and Applied Sciences, 6(1). 
Sekhar, S., \& Rao, B. C. (2011). Cottonseed Oil as Health Oil. Pertanika J. Trop. Agric. Sci., 34(1), 17-24.

Sharma, D., Pathak, D., Atwal, A. K., \& Sangha, M. K. (2009). Genetic variation for some chemical and biochemical characteristics in cotton seed oil. J. Cotton Res. Dev., 23, 1-7.

Shaver, T. N., \& Dilday. (1982). Measurement of correlation among selected seed quality factor for 36 Texas race stocks of cotton. Crop Sci., 22, 779-781. http://dx.doi.org/10.2135/cropsci1982.0011183X002200040019x

Silveira, G. D. (2007). Estimates of genetic parameters aimed selection of segregating genotypes of soybeans (Ph.D. Thesis in Genetics and Plant Breeding, State University Paulista "Julio de Mesquita Filho", Faculty of Agricultural and Veterinary Sciences UNESP in Jaboticabal, SP, p. 45).

Song, X. L., \& Zhang, T. Z. (2007). Identification of quantitative trait loci controlling seed physical and nutrient traits in cotton. Seed Sci. Res., 17, 243-251. http://dx.doi.org/10.1017/S0960258507834957

Tunc, S., \& Duman, O. (2007). Thermodynamic Properties and Moisture Adsorption Isotherms of Cottonseed Protein Isolate and Different Forms of Cottonseed Samples. Journal of Food Engineering, 81, 33-143. http://dx.doi.org/10.1016/j.jfoodeng.2006.10.015

Turner, J. H., Ramey, H. H., \& Worley, S. (1976b). Relationship of yield, seed quality, and fiber properties in Upland cotton. Crop Sci., 16, 578-580. http://dx.doi.org/10.2135/cropsci1976.0011183X001600040038x

Wakelyn, P. J., \& Wan, P. J. (2003). Extraction Optimization. In C. Tzia (Ed.), Marcel Dekker (pp. 391-427). New York.

Wan, P. J., Hron, R. J., Dowd, M. K., Kuk, M. S., \& Conkerton, E J. (1995b). Alternative hydrocarbon solvents for cottonseed extraction: Plant trials. J. Am. Oil Chem. Soc., 72, 661-664. http://dx.doi.org/10.1007/BF02635651

Wu, J., Jenkins, J. N., McCarty, J. C, \& Thaxton, P. (2009). Seed trait evaluation of Gossypium barbadense L. chromosomes/arms in a G. hirsutum L. background. Euphytica, 167, 371-380. http://dx.doi.org/10.1007/s10681-009-9896-5

Yu, J., Yu, S., Fan, S., Song, M., Zhai, H., Li, X., et al. (2012). Mapping quantitative trait loci for cottonseed oil, protein and gossypol content in a Gossypium hirsutum $\times$ Gossypium barbadense backcross inbred line population. Euphytica, 187, 191-201. http://dx.doi.org/10.1007/s10681-012-0630-3

Yunusova, S. G., Gusakova, S. D., Glushenkova, A. I., Nadzhimov, U. K., Turabekov, Sh., \& Musaev, S. A. (1991). A comparative investigation of the fatty acid compositions of the seeds of a number of lines of a genetic collection of Gossypium hirsutum. Khim. Prir. Soed., 173-176. http://dx.doi.org/10.1007/bf00629746

\section{Abbreviations}

ASE (Accelerated Solvent Extraction); GC (Gas Chromatography); THANH 100 (Techno Chroma); FID (Flame Ionization Detector); $\mathrm{NaOH}$ (Sodium hydroxide); $\mathrm{HCl}$ (Hydrochloric acid); $\mathrm{KOH}$ (Potassium hydroxide); SAS (Statistical Analysis Software); CV\% (Coefficient of Variability).

\section{Copyrights}

Copyright for this article is retained by the author(s), with first publication rights granted to the journal.

This is an open-access article distributed under the terms and conditions of the Creative Commons Attribution license (http://creativecommons.org/licenses/by/3.0/). 